\title{
How to Instantaneously Improve Your Speaking Voice
}

\author{
COMMUNICATION CORNER No. 13
}

\author{
by Philip Yaffe
}

\begin{abstract}
Editor's Introduction
Each "Communication Corner" essay is self-contained; however, they build on each other. For best results, before reading this essay and doing the exercise, go to the first essay "How an Ugly Duckling Became a Swan," then read each succeeding essay.
\end{abstract}

Although we spend much more time speaking than we do writing, the fact remains that most people speak very poorly. Phil Yaffe provides some tips on how to purposely redesign your articulation. 


\title{
How to Instantaneously Improve Your Speaking Voice
}

\author{
COMMUNICATION CORNER No. 13
}

\section{by Philip Yaffe}

Most scientists and technologists recognize the need to write well in order to better convey what they do to colleagues, managers, administrators, and the general public, but what about oral skills? After all, we spend much more time speaking than we do writing.

I am referring not only to giving formal speeches and presentations, but also informal encounters such as talking on the phone, discussing ideas with colleagues, attending meetings, participating in seminars and workshops, or simply socializing after work.

The fact is most people speak very poorly. I know, because I used to be a very poor speaker. Now I speak very well, at least that is what everyone tells me. My change from being a poor speaker to being a good speaker happened virtually overnight. I admit I had a head start because I was already a pretty good writer. I knew how to cogently organize content, but delivering it orally was quite a different matter.

Here's my story.

I was a student at UCLA (University of California at Los Angeles) in the 1960s. After graduating, I spent two years as a Peace Corps volunteer in Tanzania.

When I got there, of course everything was quite different from anything I had previously experienced. For one thing, I was posted to a tiny bush village next to what was supposed to be a major north-south road. Anything beyond 20 cars per day passing by was considered to be heavy traffic; in Los Angeles, less than 20 cars per minute was considered to be abnormally low traffic.

Perhaps the biggest surprise occurred when I turned on my shortwave radio. I quickly found the frequencies for both the BBC and Voice of America. The difference was astounding. 
Listening to the BBC, I heard cashiers, janitors, parking attendants, etc., speaking as if they had graduated from prestigious universities. By contrast, listening to Voice of America, I heard people at the top of the educational and social ladder speaking as if they had never even graduated from primary school.

This was no chance occurrence; it happened every time I turned on the radio. "How is it that high-level Americans seem to speak less well than low-level Brits?" I kept asking myself. And found no answer.

After a few weeks I came to a startling conclusion. The apparent superiority of the Brits had nothing to do with either intelligence or education. It was in fact physical.

If you pay attention, you will notice the British, particularly the English, tend to form their words on their lips, while Americans form them in the throat. This phenomenon is particularly noticeable among males, whose deep, gravely voice is considered to be masculine, virile, and seductive.

I believe forming words in the throat puts abnormal strain on the larynx, which is why Americans so often tend to stumble over their words. They frequently interrupt their speech with interjections such as "er", "um", "ah", and other irritating hesitations. This is not because they don't know what to say next. It's because they are giving their vocal cords a momentary chance to recover.

To put the idea to the test, I consciously began forming my words on my lips rather than in the throat. My own irritating hesitations (I was notorious for this) vanished almost overnight. Suddenly I could put together a sentence that flowed fluently from one end to the other. In fact, I could put together whole series of sentences that flowed fluently from one end to the other with hardly a hesitation in sight.

Having tested this discovery over several weeks, I decided to purposely redesign my articulation. It wasn't easy. I had to consciously think about how I was forming my words virtually every minute of the day. However, after a couple of weeks it began feeling more and more natural, until finally it was.

All this happened 50 years ago. During the intervening five decades, I have gained somewhat of a reputation as an entertaining and provocative public speaker, not only in English but also in French (I live in Belgium). In short, redesigning my voice has worked extremely well for me. I don't know it if would work for anyone else, but I see no reason why not.

How can you tell if you are a throat speaker or a lip speaker? Here are a couple of simple tests. 
First, put the fingers of one hand on your throat when you speak. If you can feel your vocal cords vibrating, then you are definitely a throat speaker. However, this is not definitive; you can still be a throat speaker without actually feeling the physical effect.

How?

Read a text aloud in your normal voice; then read it aloud again with a slightly higher pitch and exaggerated movements of your lips. You should hear a difference. Do this a few more times to be certain you hear a difference. Then start again with the same slightly higher pitch but less exaggerated lip movements. After a few tries, you should find a combination of pitch and lip movements that feels relatively comfortable.

From then on, each day try to use your new voice more and more frequently. It won't take too long before it will feel completely natural and you will find yourself using it all the time. I haven't used my "real" voice (i.e. the one I grew up with) in more than 50 years.

But I have two words of warning.

First, sounding better does not ipso facto make you a better speaker; it just makes you more listenable. As with writing, the real test of good speaking is what you say, i.e. content, rather than how you say it. If your ideas are ill formed and incoherent, no matter how well you express them, they will still fail to convince.

Secondly, if you do decide to make the change, you are likely to develop a pseudo "Oxbridge" English accent. This is quite normal; forming words on the lips naturally gives rise to this type of accent. However, out of context it can be rather embarrassing.

Since I was living with a lot of British expatriates in Tanzania, my new way of speaking didn't seem particularly odd. However, the minute I returned to Los Angeles, people began commenting on it, including my mother. "Where did you pick up that snooty accent? Get rid of it." So I did. I found a middle ground between the two accents. You can too.

If you are serious about improving your speaking, here's a tip. Why not join a local chapter of Toastmasters International? Toastmasters is a worldwide club of people in all walks of life dedicated to helping each other improve their orals skills. Participating is inexpensive, painless, and a lot of fun. 


\section{Mathematical Magic}

Last time, I posed the following intriguing puzzle.

A hunter leaves his cabin. He walks exactly 1 kilometer due south, then turns and walks exactly $1 \mathrm{~km}$ due east. He shoots a bear, which he drags exactly I km back to his cabin. What color is the bear?

The answer offered by the science magazine that posed the puzzle is: The bear is white.

Why? Because the Earth is a sphere, it is only at the North Pole that can you walk exactly $1 \mathrm{~km}$ due south, then $1 \mathrm{~km}$ due east, then exactly $1 \mathrm{~km}$ back to where you began. Starting at the North Pole, by definition every direction is south. If you first walk $1 \mathrm{~km}$ due south, then $1 \mathrm{~km}$ due east (or due west for that matter), you are walking along a circle, every point of which is exactly $1 \mathrm{~km}$ away from your starting point, i.e. the North Pole. Since only polar bears live around the North Polar, the beast must be white.

Ingenious, right?

Well, there is more to the story. As a perceptive reader pointed out, it is not true that the North Pole is the only point on the planet where these conditions can be fulfilled. In fact, if you start at the South Pole rather than the North Pole, there is an infinite number of points where these conditions can be fulfilled. Here's why.

1. Construct two circles, $\mathbf{A}$ and $\mathbf{B}$, around the South Pole.

2. Choose circle $\mathbf{B}$ so that its circumference is exactly $1 \mathrm{~km}$.

3. Locate circle $\mathbf{A}$ exactly $1 \mathrm{~km}$ due north of circle $\mathbf{B}$. Circle $\mathbf{B}$ is therefore exactly $1 \mathrm{~km}$ due south of circle $\mathbf{A}$.

4. Now, starting from any point on circle $\mathbf{A}$ (the northern circle), if you walk $1 \mathrm{~km}$ due south, you will always arrive somewhere on circle $\mathbf{B}$.

5. If you then walk $1 \mathrm{~km}$ due east (or due west), you will make one complete trip around the circumference circle B, i.e. $1 \mathrm{~km}$.

6. You then walk $1 \mathrm{~km}$ due north, returning you to circle $\mathbf{A}$, exactly where you started, i.e. the hunter's cabin.

In sum, rather than there being only one unique point on Earth from where you can go $1 \mathrm{~km}$ due south, then $1 \mathrm{~km}$ due east (or west), then $1 \mathrm{~km}$ back to where you started, there is an infinite number of points, i.e. all the points on circle $\mathbf{B}$ where the hunter's cabin could be located. 
Q.E.D.

Well, not quite. This rejiggering of the original solution to the puzzle breaks down in one crucial respect. There are no bears at the South Pole, so if you actually shoot a bear, the correct answer can still only be the North Pole.

But even this isn't the end of the story, or rather the end of this lesson. Robert Louis Stevenson's admonition to "Write so that you cannot possibly be misunderstood" is probably always your best guide to writing clear, concise texts. However, it is incomplete. Whenever you write, you must make certain assumptions about the knowledge and psychological status of your reader. In short, how much does your reader already know? And how is the reader likely to interpret what you say in relation of how much he already knows?

We can see this by looking at the South Pole solution to the problem in more detail.

Step 1 says to "construct two circles, A and B, around the South Pole." A moment's reflection will tell you that this is impossible. The Earth is a globe, not a plane, so any circle you construct with regard to the South Pole cannot be on a plane with the pole, but must be somewhat north of it. To be technically correct, Step 1 should read "Along the axis from the South Pole to the North Pole, construct two circles, A and B, at least 1 km north of the South Pole."

But even this wouldn't be enough because we have neglected to say that the two circles must be parallel to each other, i.e. constructed along two different lines of latitude on the globe, because only under these circumstances would walking due south from circle $A$ necessarily put you on circle $\mathbf{B}$. We assume that the reader will intuitively recognize this.

Thus, more correctly, Step 1 should read, "Along the axis from the South Pole to the North Pole, construct two circles, A and B, at least one $1 \mathrm{~km}$ north of the South Pole such that each circle is along a line of latitude." Or even more correctly, "Along the axis from the South Pole to the North Pole, construct two circles, A and B, at least one $1 \mathrm{~km}$ north of the South Pole such that each point on circle $\mathbf{A}$ is exactly $1 \mathrm{~km}$ from a corresponding point on circle $\mathbf{B}$ and that each circle is along a different line of latitude."

But once again, we are not quite finished. In this version of the instruction, we are assuming that the reader instantly grasps the significance of the circles being constructed along "lines of latitude," which is probable but not certain.

Therefore, to be even more correct, Step 1 should probably read: "Along the axis from the South Pole to the North Pole, construct two circles, $\mathbf{A}$ and $\mathbf{B}$, at least one $1 \mathrm{~km}$ north of the 
South Pole such that each point on circle $\mathbf{A}$ is exactly $1 \mathrm{~km}$ from a corresponding point on circle B and each point on circle A is equidistant from the South Pole, as is each point on circle B."

Tedious, isn't it? Worse, framing the instruction this way makes picturing the situation in one's mind eye rather more difficult. If each step in the solution were written with such precision in order to obviate assumptions, the chances of anyone understanding the solution would become vanishingly small.

The moral of the story: Write so that you cannot possibly be misunderstood. However, closely inspect the assumptions you are making in trying to avoid being misunderstood. As with the bowls of porridge in the fairy-tale "Goldilocks and the Three Bears," do not make too many assumptions or too few, but get it just right.

As a footnote, a fellow Ubiquity editor made a comment about the bear puzzle that I had not previously seen when I visited the many that talked about it. He shared the following:

"We see there are two places on Earth where a hunter could make the journey specified in the puzzle. The north hunter's cabin is directly on the pole, and the south hunter's cabin is on circle $\mathbf{A}$. For both hunters, the number of possibilities is infinite. The north hunter can start his journey facing any way he wishes, because by definition his first step will always be south, while the south hunter can locate his cabin at any point on circle $\mathbf{A}$ he wishes. Mathematically, we can say there are two infinite families of hunter journeys. The puzzle writer had to help the reader select one of the two. That is where the polar bear came from."

\section{HOMEWORK: Retrospective to Communication Corner No. 12}

There was no formal homework for Communication Corner No.12. You were simply asked to pay attention to how often people needlessly use um, ah, like, you known, and other useless sounds and how annoying they can be. I hope you did so and are now convinced of the imperative need to eliminate them as much as possible. Learning how to do so was the purpose of this current "Communication Corner" essay.

\section{CURRENT HOMEWORK}

This will be perhaps the easiest-and hardest-homework assignment of this series, because it is divided into two exercises. 


\title{
Exercise 1: Listen and Learn
}

Closely listen to any speaker you admire (politician, business leader, celebrity, educator, friend, etc.) in an impromptu situation, and ask yourself why you admire him or her. I specify an impromptu situation (i.e. interview, news conference, company meeting, etc.) because this is where they really shine. They are required to speak largely off the cuff. They haven't spent hours of preparation as with a formal speech, yet sometimes it seems as if they have because there is hardly an um, ah, like, you know in earshot.

\section{Exercise 2: Try It Yourself}

Now it's your turn. Pay close attention to how you speak in impromptu situations, consciously trying to avoid filler words. At first, this may seem difficult because using filler words is "natural," except that it isn't. Remember the advice from Toastmasters.

For any subject, there are usually several ways of saying the same thing. If you are always looking for the "best way" (assuming there is one), then you will invariably find yourself inserting "ums", "ahs", "you knows", and other distractions. In the vast majority of cases, sacrificing fluency while searching for the best word or words just isn't worth it. So go with what you have.

If something comes out of your mouth that you think you could have said better, simply start your next sentence with, "To be more precise. . ..", then say it better. This technique will not only keep your speech fluent, it will make you appear to be master of your subject, not its apprentice. What could be better than that?

In short, don't worry about it. Just do it.

\begin{abstract}
About the Author
Philip Yaffe was born in Boston, Massachusetts, in 1942 and grew up in Los Angeles, where he graduated from the University of California with a degree in mathematics and physics. In his senior year, he was also editor-in-chief of the Daily Bruin, UCLA's daily student newspaper. He has more than 40 years of experience in journalism and international marketing


communication. At various points in his career, he has been a teacher of journalism, a reporter/feature writer with The Wall Street Journal, an account executive with a major international press relations agency, European marketing communication director with two major international companies, and a founding partner of a specialized marketing communication agency in Brussels, Belgium, where he has lived since 1974. He is the author of more than 20 books, which can be found easily in Amazon Kindle.

DOI: $10.1145 / 3319332$ 